\title{
Development and implementation of new principles and systems for train traffic control in Sweden
}

\author{
B. Sandblad, A. W. Andersson, A. Kauppi \& G. Isaksson-Lutteman \\ Human-Computer Interaction, Dept of Information Technology, \\ Uppsala University, Sweden
}

\begin{abstract}
The trend towards higher speed, more frequent traffic and many traffic operators requires new strategies and solutions for efficient train traffic control and utilization of track capacity. Operative control is today focused on controlling the infrastructure. In earlier research we have shifted the control paradigm from today's technology oriented into a more traffic oriented one. This is done by real-time re-planning. The continuously updated traffic plan is normally executed by automated systems. After tests and evaluation in a simulated laboratory environment, the Swedish Rail Administration (Banverket) decided to develop and deploy an operative system to be installed at a traffic control centre. This system, called STEG, implements the main research results. Features of the new system are a dynamic planning view in form of a time-distance graph, decision support that helps the controller to identify disturbances and conflicts and automatic systems for execution of the traffic plan. The traffic controller can re-plan traffic (time aspects, track usage) via direct manipulation of the graph lines in the interface. Track maintenance and other activities can also be planned. The system automatically calculates all consequences of the changes and shows the effects on all trains within the actual time-distance space. A very careful process has been used to go from research results and prototypes to a fully operational system. The process has been very user centred and numerous iterations have been performed. Through this elaborate work, we have been able to ensure that the intentions of the prototypes have been correctly implemented in the final product.
\end{abstract}

Keywords: train traffic control, dispatching, traffic planning, user interfaces, automatic execution. 


\section{Background}

Tomorrow's train traffic, with higher speed, more frequent traffic, mixed traffic and many independent traffic operation companies, requires new principles and technical solutions for efficient train traffic control. Today's control systems are often designed to support the operator's possibilities to react on alarms, conflicts and disturbances and to solve acute problems and conflicts. However, in order to perform efficiently, operators should be able to follow the dynamic development of the traffic system over time and prevent disturbances. In order to achieve this, we must change the control paradigm from technical control of the infrastructure into higher level traffic planning tasks. This is done by replacing the traditional control commands by real-time re-planning (Andersson et al. [1], Sandblad et al. [7], Wikström et al. [9]).

Advanced laboratory prototypes have successively been implemented and tested. By connecting user interface prototypes to a train traffic simulator (Sandblad et al. [8]), it has been possible to perform experiments with the design of new user interfaces and decision support tools, and to test and evaluate new control strategies for the train traffic control operators. Based on numerous laboratory experiments, a step has now been taken in order to build a fully operational system and to test and evaluate this in a real traffic control centre environment.

\section{Earlier research studies}

Our research has been based on a very detailed description and analysis of how train traffic is controlled today, the mental models of the dispatchers and the strategies they use for decisions and control tasks. The research has consisted of mainly the following steps:

- Observations and interviews with dispatchers and other professionals at the traffic control centres. Analysis of the findings and identification of problems and development areas.

- Seminars with experienced and responsible professionals from the national rail and traffic control administrations. Here the visions and restrictions for future development of control systems have been specified.

- Iterative specifications and evaluations with the help of a working group consisting of experienced operative traffic control professionals.

- $\quad$ Tests and evaluations in a laboratory control room environment using a train traffic simulator system.

In order to support real-time planning of train traffic we provide the traffic controller with an interactive computerized time-distance graph. Prototypes of new user interfaces that support the new control strategy have been designed, implemented and preliminary tested in the laboratory environment at Uppsala University. The interface is designed to integrate all decision relevant information into one unified interface and to support continuous awareness of the dynamic development of the traffic process (Kauppi et al. [5]). 
The computer based time-distance graph is designed in such a way that it visually supports the operators' situation awareness of the current status and the projection into the future (Endsley [3]). The user interface, with its planning view, can support early detection of upcoming conflicts, identify possible replanning alternatives and their predicted effects. The new control strategy has a potential to better support the traffic controller's ability to handle continuous replanning, with the goal to always have a functional traffic plan at hand. This plan can be automatically executed except when technical malfunctions hinder this. Automatic functions that support execution of the traffic plan must be transparent, predictable and easy to understand. The automatic functions must never change the controllers' traffic plan but are only allowed to strictly execute the actual traffic plan. The traffic plan mainly consists of time table and track usage information, including maintenance work. Detailed interface design, easy to interpret, concerning the automation helps to keep the human in-the-loop and to avoid automation surprises (Bainbridge [2]). By re-planning, the operator is in control of what the automatic function will do and when. Hence, the operator is continuously in full and active control.

We have also evaluated different approaches to include decision support systems in operative train traffic control (Hellström et al. [4], Kvist et al. [6]). We have found that more advanced automated decision support systems are today not a realistic alternative of several reasons. More research and development of methods are needed in this field. We have decided to focus our efforts on supporting the controllers through better presentation of information, improved information observability and quality, help with early detection of conflicts and disturbances, identification of possibilities and limitations for replanning and evaluation of effects of alternative actions.

\section{From research prototypes to an operational system}

Experiments with the new control strategy, operator interfaces, decision support systems and automatic execution functions have been performed in our laboratory environment with satisfying results (Sandblad et al. [10]). Many important aspects can be investigated in the simulated environment at Uppsala University, but some issues must be evaluated in a real operative environment. To work in a laboratory environment, and to control a simulated traffic system, will always mean that we have simplified the situation. The real traffic system is more complex and stochastic compared to our laboratory models. The work tasks of the controllers are also more complex and diversified then what we can create in the laboratory, e.g. concerning communication with other persons in the complex and dynamic environment. It will never be possible to evaluate all relevant aspects of the new control system in a pure simulated environment.

We also face large practical and economic problems when the laboratory prototypes shall be implemented and deployed as a part of the real train traffic system. It will not be possible to develop a complete traffic management and control system only for test purposes, but we must implement the new control functionalities on top of the existing basic control infrastructure. Our prototype 
system has a focus on planning, re-planning and automatic execution of control commands. Not all other tasks are supported by functionality in our system. When malfunctions in the infrastructure hinder the automatic execution, the controllers must go back to the old control system. Thus, the new control system, control by re-planning, must for test purposes be implemented as a complementary module to the existing system.

The research and technical implementation questions that we try to answer in the project are mainly:

- Does the new control paradigm, principles, tools and interfaces really contribute to more efficient traffic control and a better work environment for the traffic controllers?

- Is it possible to implement the new control principles and tools as an integrated part of the already existing infrastructure? What of the original ideas must be changed in order to make the implementation possible and economically realistic?

- How can our research prototype support requirement specifications and evaluation for the implementation and development process?

\section{The STEG project}

\subsection{Project phases}

The STEG project has been divided into several different steps or phases. On a high level the following main steps have been identified:

- Benefit-cost analysis.

- Risk analysis and assessment of the project as such, including backing procedures if certain parts of the project fail.

- Identification of test site. A test site was selected that fulfilled a number of requirements, e.g. availability of different track structures, single track, double track, mixed traffic types, more complex stations, connections to other traffic control regions, availability of input data for track diagrams, technical specifications etc.

- Requirement specification for the test system.

- Several different technical investigations concerning compatibility, availability of input data, technical platform, technical performance, safety, security, communication etc.

- Specification and test of control algorithms, e.g. for the automatic execution of control commands from the traffic plans.

- Technical development according to specified development model, including a user centred process.

- Implementation. Operative tests and evaluation.

\subsection{The development phase}

Of special interest in a research context is the process to come from the research prototype to a fully operational system without loss of essential requirements and 
functionality. This has successfully been achieved through a very close cooperation between researchers, designers and developers, including numerous iterations. We have earlier found that it is not so easy to create a system that to full extent meets all relevant requirements.

In this project there has been a very strong focus on usability issues, minimizing the operator's mental workload, support of the operator's situation awareness, avoiding automation surprises etc. In order to achieve all this in an optimal way, it is not possible to supply the technical development team with a comprehensive list of requirements, and passively wait for the final system. Every step in the development process must be followed, analysed and evaluated by skilled interface designers with a deep knowledge in appropriate knowledge areas. Of course, this requires both enough time and resources together with a development team that is open to continuous iterations, tests and modifications.

\section{The STEG system today}

The STEG system is today implemented as an additional module on top of the regular train traffic control system. This allows the traffic planner to go back to the old traditional system at any time. Via STEG, the traffic planner can continuously observe the dynamic development of the traffic within the actual track segment. The planning view in the time-distance graph is automatically scrolled downwards as time evolves. Identified conflicts with respect to track usage on the train lines or in the stations are automatically indicated in the interface. Such conflicts can now be early identified and eliminated by the traffic planner by re-planning of time table and track usage for each train involved in the conflict. Other sets of information shown in the interface are track structure, train positioning, detailed information concerning trains and stations etc. The user interface is continuously updated by dynamic data from the train traffic and signalling system. The results of all re-planning actions and the total effects of the valid traffic plans are always shown in the interface. See figure 1.

Through manipulation of the time-distance graph lines, directly in the user interface, the time-table and the track usage can be re-planned whenever the traffic planner finds this appropriate. See figure 2. When the traffic plans are without conflicts, they can be automatically executed. This is done by a separate system that executes the plans exactly as they are specified by the traffic planner. By not allowing the automatic algorithms to change the traffic plans, all "automation surprises" (Bainbridge [2]) can be eliminated. The automatic execution system is "non-autonomous" and is never allowed to change the traffic plan. In earlier systems, where the automatic systems could change the plan, train order, meeting-points etc., the traffic planner often turned the automatic system off in order to avoid confusion. The human traffic planner is now always in full control of the situation. 


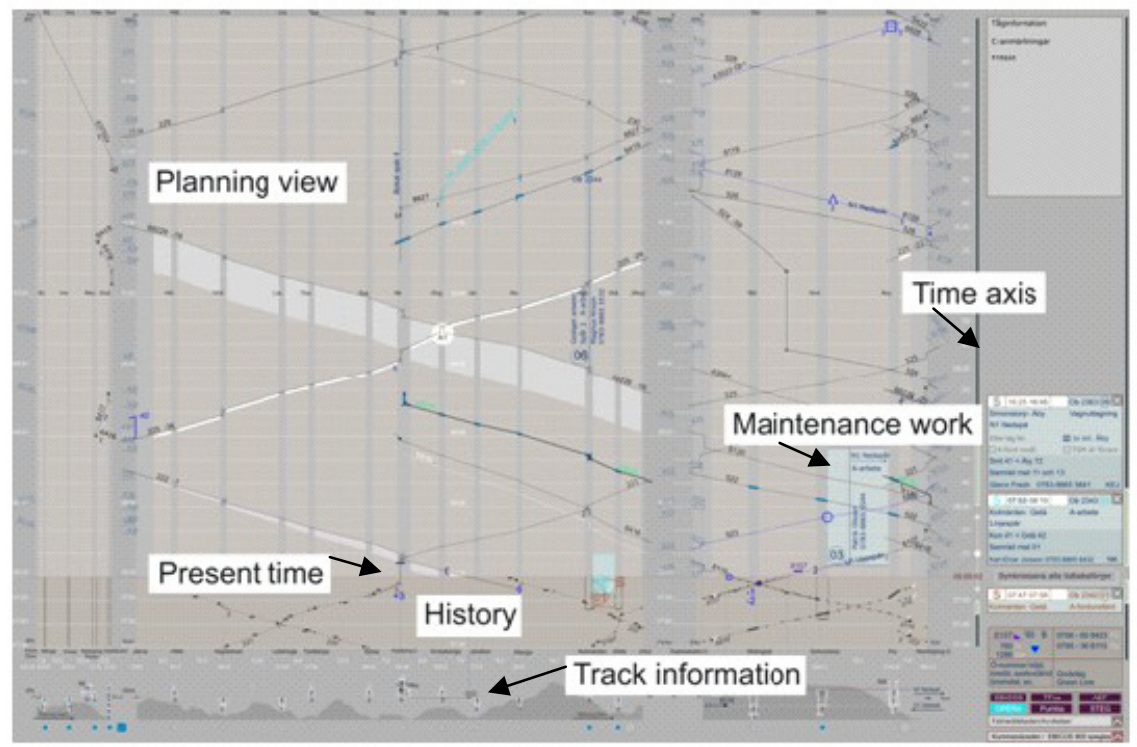

Figure 1: The figure shows the united interface, including the planning view in the time-distance graph, graph lines for each individual train, the time axis, the history below the present time line, the track structure, train and station information, planned maintenance work, etc. The planned traffic can always be seen together with the original time-table lines, so that delays, etc. can be easily detected. Conflicts of different natures are also visualized and can be detected and solved early.

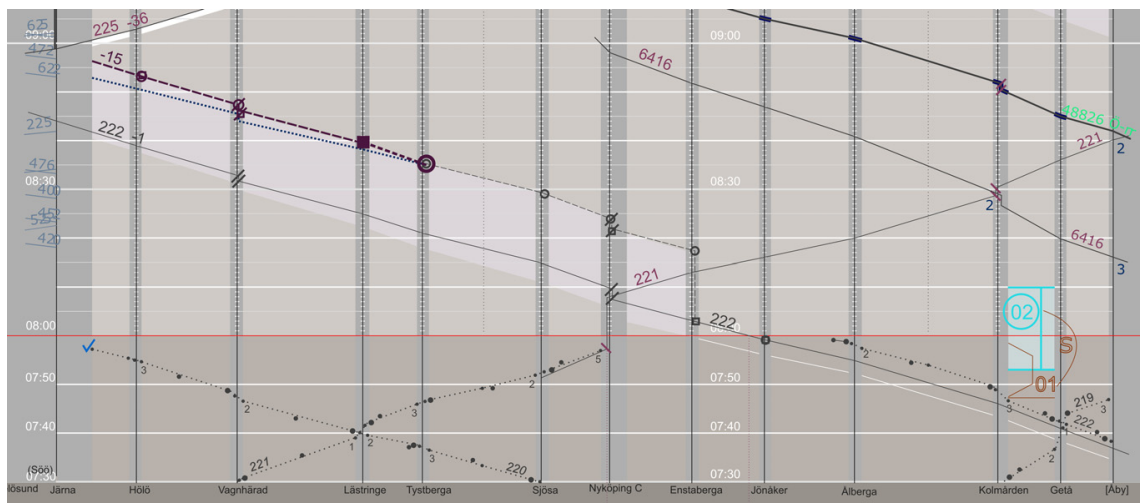

Figure 2: The figure shows re-planning of a selected train. The traffic planner can easily change the arrival time, departure time or track usage for the selected train and station. This is done by the selection of a graph line and manipulation of the nodes using the mouse buttons and thumb-wheel. The new plan can be seen directly in the user interface. 


\section{Preliminary tests and evaluation}

The STEG system has been used for operational tests at a Swedish train traffic control centre. Via STEG, traffic planners can monitor and control by replanning one segment of the main rail line from Stockholm to southern Sweden, including one double-track line, one single-track line and some less frequently used freight traffic lines.

The experiences so far, when the test period is not finished, are mixed. On one hand, the system works according to the intentions and requirements. On the other hand, a number of different problems have appeared which have made the tests somewhat problematic.

We have found that the basic concept, control by re-planning in real time and automatic execution of the traffic plans, is working in practice and is accepted by the traffic planners. However, we have also been faced with a number of problems of a more practical nature. Some of the more important problems and obstacles, which have disturbed the tests so far, are:

- Technical errors in old interlocking systems, difficult and expensive to eliminate.

- The user interface should show more relevant states of the automatic execution system. Otherwise the traffic planner will not be able to predict the effects of technical malfunctions.

- A larger presentation area would improve the usability.

- Lack of a complete integration with the ordinary traffic control and signalling system leads to robustness problems.

More advanced tests and evaluation procedures are planned for the remaining test period. These include e.g. data analysis, observations, interviews, questionnaires and video recordings with following analysis of the planners' behaviour. The evaluation will focus on two main questions: does the system contribute to better traffic performance and does the system contribute to more efficient work of the traffic planners. The full results of the evaluation will be presented later.

\section{Future research}

From our earlier research and experimental studies in the laboratory, which has been shortly discussed above, we have a more or less complete solution for the new proposed control paradigm, control by re-planning. When the operative test system is being specified and developed, it is not possible to implement the full prototype system. Some parts are not relevant to the operative test environment, other parts are not possible to implement because of limitations in the existing infrastructure etc. Some of the more important, and from a research point of view most interesting, problems to be solved in the future are: 


\subsection{Traffic planning on a national level}

It is not enough to solve the re-planning problem for individual traffic segments. In Sweden today the traffic is controlled as several isolated traffic segments in each of the eight regional control centres. In order to obtain continuously updated traffic plans that cover the total traffic system, a national system and organisation must be created that integrates all separate traffic plans. On this national level more strategic planning decisions can be taken, which better coordinates the local and regional activities.

\subsection{Automatic execution functions}

The purpose of the automatic execution functions is to generate and deliver control command sequences to the underlying control system in time. Because of the lacking quality in traffic predictions, the algorithms must have large margins. This results in a non optimal performance. Measures to improve precision in data are most important, since this can significantly improve the total performance. The traffic controllers could e.g. be allowed to update the traffic plans closer to real-time. The actions of the automatic functions must be clearly shown in the user interface in order to support good situation awareness and avoid automation surprises.

\subsection{Detailed track diagrams}

Today it is unclear how much track diagram information that is needed for the controllers. The presentation must be detailed enough to support the understanding of conflicts, status of the infrastructure, restrictions and degrees of freedom in the re-planning activities etc. Different level of detail in the presentation will be tested in the future.

\subsection{Traffic control in complex stations}

The design of support systems, e.g. interface elements and decision support functions, for traffic control in more complex stations is not investigated enough. In our operative test environment we will not cover complete traffic regions, and because of that we do not now need advanced solutions. On the other hand, we will not be able to evaluate the total performance. In order to specify a complete traffic plan, from start to end station, also complex stations must be covered by the re-planning tool.

\subsection{Work environment and design of the workplace}

We have a rather detailed picture of what kind of presentation system that is needed for optimal performance and a good work environment. This should require very large presentation areas with high resolution and quality and without disturbing frames. Because of economic and practical reasons, we will not be able to implement an ultimate technology. The exact lay-out of the work place 
will be made so that the individual traffic controller has an optimal solution concerning ergonomics and information overview.

\subsection{Remaining design and implementation questions}

There are some important problems concerning the available technical solutions that we will not be able to solve within the STEG project. This will restrict our possibilities to develop efficient support systems and to evaluate the new control paradigm. The two most severe limitations in this respect are:

- Train speed and position. There are no technical solutions available that now allow us to observe train speed and position with high precision. Today the best precision is the identity of the block section. In the future positioning systems with a high precision will be available.

- Communication with train drivers. Today we are not able to automatically communicate new traffic plans to the train drivers. This means that the train drivers will drive according to old and obsolete plans. By doing so they will not be able to perform optimally. In very urgent cases the controllers can phone the train drivers to inform them about changes in traffic plans, train stops etc. It is also not possible for the train drivers to easily inform the traffic controllers about late departures, speed restrictions caused by machine problems etc. In the future we will have efficient communications links for such purposes, e.g. when ERTMS/ETCS systems have been fully implemented.

\section{Acknowledgements}

This project has been financially supported by the Swedish National Rail Administration. We especially thank all professional traffic controllers and planners who have been engaged in the research and test activities.

\section{References}

[1] Andersson A.W., Sandblad B., Hellström P., Frej I., Gideon A. (1997) A systems analysis approach to modelling train traffic control. Proceedings of WCRR'97, Florence, Italy 1997.

[2] Bainbridge, L. (1983). Ironies of automation. Automatica, 19, 775-779.

[3] Endsley M.R. (1996). Automation and situation awareness. In R. Parasuraman \& M. Mouloua (Eds), Automation and Human performance: Theory and applications (pp. 163-181). Mahwah, NJ: Lawrence Erlbaum.

[4] Hellström, P., Sandblad, B., Frej, I., Gideon, A. (1998). An evaluation of algorithms and systems for Computer-Aided Train Dispatching, Computers in Railways VI, Wessex Institute of Technology, 1998.

[5] Kauppi A., Wikström J., Hellström P., Sandblad B., Andersson A. W., (2005). Future train traffic control, control by re-planning. In J.R. Wilson, B. Norris, T. Clarke, and A Mills (Eds), Rail Human Factors supporting the integrated railway (pp. 296-305). Ashgate Publ. Ltd. 2005. 
[6] Kvist T., Hellström P., Sandblad B., Byström J., (2002). Decision support in the train dispatching process. Computers in Railways VIII, Wessex Institute of Technology, 2002.

[7] Sandblad B, Andersson AW, Byström J, Kauppi A. (2002). New control strategies and user interfaces for train traffic control. Computers in Railways VIII, Wessex Institute of Technology, 2002.

[8] Sandblad B. et al. (2000). A train traffic operation and planning simulator. Computers in Railways VII, Wessex Institute of Technology, 2000.

[9] Wikström J., Kauppi A., Hellström P., Andersson A., Sandblad B. (2004) Train traffic control by re-planning in real-time. Computers in Railways IX, Wessex Institute of Technology, 2004.

[10] Sandblad, B., Andersson, A.W., Kauppi, A. and Wikström, J. Implementation of a Test System for Evaluation of New Concepts in Rail Traffic Planning and Control. In: Wilson, J., Norris, B., Clarke, T. and Mills, A. eds.: People and Rail Systems. Human Factors at the Heart of the Railways. Ashgate Publ. Comp., 2007 